\title{
Hypolipidemic effect of SR-BI gene delivery by combining cationic liposomal microbubbles and ultrasound in hypercholesterolemic rats
}

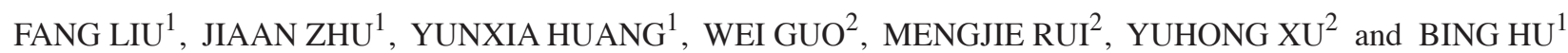 \\ ${ }^{1}$ Department of Ultrasound, Shanghai Jiaotong University Affiliated Sixth People's Hospital, \\ Shanghai Institute of Ultrasound in Medicine, Shanghai 200233; \\ ${ }^{2}$ Pharmacy School of Shanghai Jiao Tong University, Shanghai 200233, P.R. China
}

Received December 21, 2012; Accepted April 12, 2013

DOI: $10.3892 / \mathrm{mmr} .2013 .1438$

\begin{abstract}
High-density lipoprotein (HDL) is a key mediator in reverse cholesterol transport and is involved in a mechanism known as 'selective lipid uptake', a process mediated by scavenger receptor B type I (SR-BI), which is a HDL receptor. The aim of the present study was to investigate the therapeutic effect of the SR-BI gene when delivered by combining cationic liposomal microbubbles (CLMs) and ultrasound (US) in hypercholesterolemic rats. Hypercholesterolemia was induced by administration of excessive doses of vitamin D3 and cholesterol in rats. The CLMs consisted of perfluoropropane gas encapsulated in a phospholipid shell using the sonication-lyophilization method. The SR-BI gene, mixed with the self-made microbubbles, was transfected into hypercholesterolemic rat arteries using therapeutic US. SR-BI protein expression was determined by western blot analysis 2 days post-transfection. Two weeks after transfection, total cholesterol (TC), triglyceride (TG), low-density lipoprotein (LDL) and HDL serum concentrations were measured. Transfection efficiency of the SR-BI gene in the SR-BI + US/CLM group increased 6-7-fold compared with the SR-BI group. Two weeks after transfection, plasma lipid levels in treated hypercholesterolemic rats were observed to be significantly reduced compared with rats that did not receive treatment. However, no significant change was observed in the SR-BI group compared with that in the SR-BI + US/CLM group. Results of the present study indicate that the combination of US and CLMs loaded with the SR-BI gene may exert a protective role in hypercholesterolemia.
\end{abstract}

Correspondence to: Professor Jiaan Zhu, Department of Ultrasound, Shanghai Jiaotong University Affiliated Sixth People's Hospital, Shanghai Institute of Ultrasound in Medicine, 600 Yishan Road, Shanghai 200233, P.R. China

E-mail: zhuja@sjtu.edu.cn

Key words: hypercholesterolemia, scavenger receptor B type I, ultrasound, microbubble, gene therapy, rat

\section{Introduction}

Cardiovascular disease, a leading cause of mortality in developed countries, is a chronic disease that remains asymptomatic for decades. The incidence rate of this disease is increasing worldwide. Hypercholesterolemia is a major risk factor for the development of cardiovascular disease and modern lifestyles, consisting of high-cholesterol diets and less physical activity, contribute to hypercholesterolemia, which increases the prevalence of cardiovascular disease (1). Epidemiological and interventional studies have reported that high density lipoprotein (HDL) may be protective by reversing cholesterol transport, inhibiting the oxidation of low density lipoprotein (LDL) and neutralizing the atherogenic effects of oxidized LDL (2). HDL has been hypothesized to protect against atherosclerosis through its involvement in a reverse cholesterol transport pathway $(3,4)$. The scavenger receptor B type I (SR-BI), a functional cell surface HDL receptor, binds HDL and mediates efficient lipid uptake, and may be important for HDL metabolism (5).

Progression in gene therapy has led to the development of a novel treatment strategy for cardiovascular diseases. Successful gene therapy depends on the design of efficient, safe and stable gene delivery systems. Viral and non-viral vectors have been used for DNA delivery with varying levels of success, but have always been accompanied by disadvantages, including insufficient expression levels or safety concerns $(6,7)$. In previous years, the use of microbubbles and ultrasound (US) has been proposed for gene delivery. Microbubble-enhanced US alters cell membrane permeability for a short time due to sonoporation, which allows extracellular macromolecules, including plasmid DNA, to instantaneously enter cells without causing cytotoxicity (8-12). This technique has been applied to the site-specific intracellular delivery of macromolecules in vitro and in vivo $(13,14)$. Cationic lipids are associated with specific advantages as gene delivery carriers $(15,16)$. Therefore, we hypothesized that cationic liposomal microbubbles (CLMs) may represent novel gene delivery agents.

Based on cationic lipid technology, microbubbles were developed using the sonication-lyophilization method. The physiochemical properties of CLMs were analyzed to deter- 
mine their suitability as a contrast agent in medical imaging with US. In addition, the efficiency of gene transfer and the hypolipidemic effect of the SR-BI gene were evaluated using US in rats.

\section{Materials and methods}

Plasmid purification and microbubble preparation. Full-length cDNAs of the rabbit SR-BI gene were subcloned into the pcDNA3.1 vector (Invitrogen Life Technologies, Carlsbad, CA, USA) as previously described (17). Plasmid DNA amplification was performed using the Qiagen plasmid Giga kit (Qiagen, Germany).

Cationic liposomes composed of 1,2-dipalmitoyl-sn-glycero-3-phosphocholine (NOF Corporation, Tokyo, Japan), 1,2-distearoyl-sn-glycero-3-phosphoethanolamine-N-[methoxy(polyethylene glycol)-2000] (Avanti Polar Lipids, Inc., Alabaster, AL, USA) and 1,2-dioleoyl-3-trimethyl-ammonium-propane [94:6:100 (m/m)] were constructed using the sonication-lyophilization method. Mixed components were dissolved in an appropriate volume of tertiary butyl alcohol. After the components were dissolved, sonication was performed at $30^{\circ} \mathrm{C}$ (frequency, $40 \mathrm{kHz} ; 5 \mathrm{~min}$ ). The microparticle suspension solution was stored at $0^{\circ} \mathrm{C}$ for $30 \mathrm{~min}$ and $-20^{\circ} \mathrm{C}$ for $1 \mathrm{~h}$. Next, the coagulated solution was lyophilized at $5 \times 10^{-4} \mathrm{~Pa}$ pressure for $20 \mathrm{~h}$ (primary drying at $-48^{\circ} \mathrm{C}$ for $15 \mathrm{~h}$ and gradual increases in temperature to $10^{\circ} \mathrm{C}$ within $5 \mathrm{~h}$ ). Lyophilized powder was placed in $10-\mathrm{ml}$ penicillin vials for subsequent analyses. Vials were removed from the freeze dryer, flushed with perfluoropropane gas and the vial stoppers were fully closed and sealed with crimp aluminum caps. Following this, $5 \mathrm{ml}$ PBS solution was added to the lyophilized powder, followed by gentle shaking to form an emulsion-like solution. The size distribution and Zeta potential measurements of the microbubbles were measured with a Zetasizer Nano S (Malvern Instruments, Malvern, UK) after the samples were diluted with water. Each sample was measured four times and the results were expressed as the average \pm SD of three samples with a corresponding polydispersity index (PDI) value that was indicated for the size distribution measurements.

SR-BI DNA was added to the pre-formed microbubble solutions and gently agitated. SR-BI DNA was then combined with CLMs by electrostatic charge coupling.

Experimental animals. Seventy male Sprague-Dawley rats (weight, 250-300 g) were used in this study. Rats were supplied by the Experimental Animal Center of Shanghai Jiaotong University Affiliated Shanghai Sixth People's Hospital (Shanghai, China) and the experimental protocols were approved by The Animal Care and Use Committee of the Shanghai Jiaotong University Affiliated Shanghai Sixth People's Hospital.

Hypercholesterolemic animal model. The experimental hypercholesterolemic model was established in rats by administration of excessive vitamin D and cholesterol. Rats were randomly divided into the control $(n=20)$ and model $(n=40)$ groups. In the model group, rats were intraperitoneally injected with $600,000 \mathrm{IU} / \mathrm{kg}$ vitamin D3, followed by an additional $300,000-\mathrm{IU} / \mathrm{kg}$ dosage, which was repeated every 30 days.

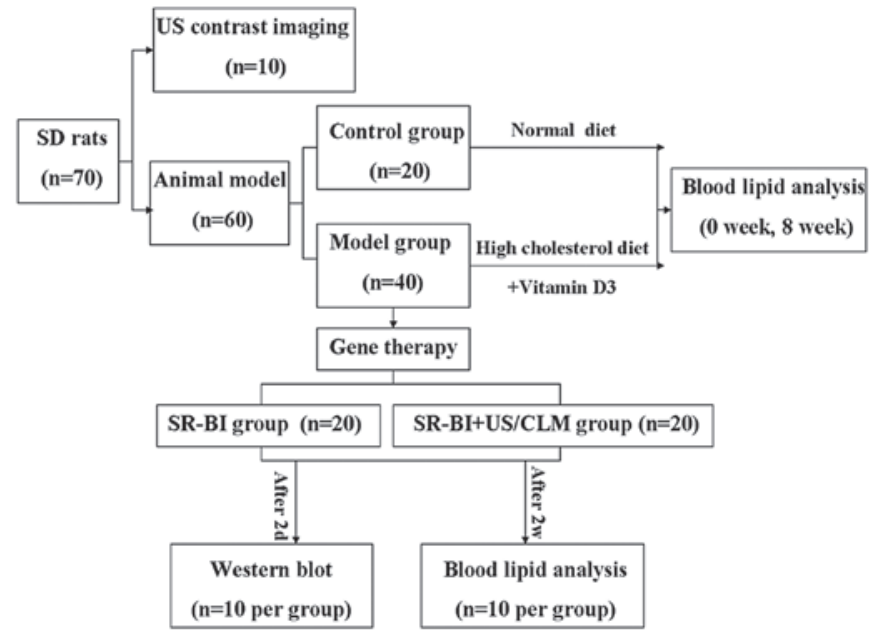

Figure 1. Schematic diagram of the experimental animal protocol. US, ultrasound; SD, Sprague-Dawley; CLM, cationic liposomal microbubbles; SR-BI, scavenger receptor B type I.

During model development, rats were fed a high-cholesterol diet daily. Blood lipid analysis was performed at week 0 (baseline) and 8 weeks following the high cholesterol diet (Fig. 1). Blood samples were collected in chilled heparinized centrifuge tubes by cardiac puncture with heparinized syringes and centrifuged at $4^{\circ} \mathrm{C}$ for $15 \mathrm{~min}$. Collected plasma samples were stored at $-80^{\circ} \mathrm{C}$ until further analysis. The total plasma cholesterol (TC), triglyceride (TG), LDL and HDL levels were measured using reagents from Nanjing KeyGen Biotech Co., Ltd. (Nanjing, China).

US contrast imaging following intravenous injection of microbubbles. Ten rats were anesthetized with $4 \%$ pentobarbital sodium $(1 \mathrm{ml} / \mathrm{kg})$ by intraperitoneal injection, placed on their backs and restrained on an experimental table. Prior to the experiments, the femoral vein was catheterized and the hair over the neck was removed with depilatory cream.

An ultrasonic diagnostic instrument (Mylab. 90; Esaote, Genoa, Italy) with $13-\mathrm{MHz}$ linear-array transducers was used. As the carotid artery was clearly depicted, all conditions discussed were kept consistent during contrast imaging.

CLMs were injected via the femoral vein at a dose of $0.5 \mathrm{ml} / \mathrm{kg}$, followed by normal saline to wash the tube. The bolus injection of microbubbles was flushed into the circulation of the animal and was favorable for improving the signal-to-noise imaging ratio. Real-time imaging of the carotid artery was observed for 5 min following injection. Images were saved during enhanced ultrasonography.

In vivo gene delivery and biochemical assay. Forty rats were anesthetized and handled as described. CLMs were injected slowly via the femoral vein within the specified time.

Gene delivery was then performed according to the assigned treatment group: SR-BI (local injection of $100 \mu \mathrm{g}$ SR-BI); SR-BI + US/CLM (intravenous push of $100 \mu \mathrm{g}$ SR-BI); and CLM ( $1 \mathrm{ml}$ ) by US ( $\mathrm{n}=20 /$ group). US parameters were as follows: frequency, $1 \mathrm{MHz}$; duty, $50 \%$; intensity, $2.0 \mathrm{~W} / \mathrm{cm}^{2}$; and time, $15 \mathrm{~min}$. Rats ( $\mathrm{n}=10 /$ group) were sacrificed 2 days post-transfection. Tissue was obtained for western blot 
Table I. Plasma lipid levels of hypolipidemic rats induced by vitamin D3 administration and a high cholesterol diet.

\begin{tabular}{|c|c|c|c|c|c|c|c|c|}
\hline \multirow[b]{2}{*}{ Group } & \multicolumn{2}{|c|}{$\mathrm{TC}(\mathrm{mmol} / \mathrm{l})$} & \multicolumn{2}{|c|}{$\mathrm{TG}(\mathrm{mmol} / \mathrm{l})$} & \multicolumn{2}{|c|}{$\mathrm{LDL}(\mathrm{mmol} / \mathrm{l})$} & \multicolumn{2}{|c|}{$\mathrm{HDL}(\mathrm{mmol} / \mathrm{l})$} \\
\hline & 0 weeks & 8 weeks & 0 weeks & 8 weeks & 0 weeks & 8 weeks & 0 weeks & 8 weeks \\
\hline Control & $0.96 \pm 0.05$ & $1.90 \pm 0.02$ & $0.31 \pm 0.02$ & $0.33 \pm 0.05$ & $0.28 \pm 0.05$ & $0.34 \pm 0.01$ & $1.12 \pm 0.03$ & $1.09 \pm 0.02$ \\
\hline Model ( $\mathrm{n}=40)$ & $1.12 \pm 0.05$ & $2.91 \pm 0.05^{\mathrm{a}}$ & $0.30 \pm 0.01$ & $1.94 \pm 0.10^{\mathrm{a}}$ & $0.29 \pm 0.07$ & $0.72 \pm 0.09^{\mathrm{a}}$ & $1.10 \pm 0.05$ & $0.48 \pm 0.02^{a}$ \\
\hline
\end{tabular}

Data are presented as the mean $\pm \mathrm{SD}$. ${ }^{\mathrm{a}} \mathrm{P}<0.05$ vs. control. TC, total cholesterol; TG, triglyceride; LDL, low density lipoprotein; HDL, high density lipoprotein.

Table II. Plasma lipid levels of rats in the model, SR-BI and SR-BI + US/CLM groups.

\begin{tabular}{|c|c|c|c|c|}
\hline Group & $\mathrm{TC}(\mathrm{mmol} / \mathrm{l})$ & TG (mmol/l) & $\mathrm{LDL}(\mathrm{mmol} / \mathrm{l})$ & $\mathrm{HDL}(\mathrm{mmol} / \mathrm{l})$ \\
\hline Model $(n=40)$ & $2.91 \pm 0.05$ & $1.94 \pm 0.10$ & $0.72 \pm 0.09$ & $0.48 \pm 0.02^{\mathrm{b}}$ \\
\hline SR-BI $(n=10)$ & $2.53 \pm 0.03$ & $1.78 \pm 0.21$ & $0.63 \pm 0.05$ & $0.75 \pm 0.10$ \\
\hline SR-BI + US/CLM $(n=10)$ & $2.36 \pm 0.11^{\mathrm{a}}$ & $0.61 \pm 0.09^{\mathrm{a}, \mathrm{b}}$ & $0.46 \pm 0.04^{\mathrm{b}}$ & $0.90 \pm 0.07^{\mathrm{a}, \mathrm{b}}$ \\
\hline
\end{tabular}

Data are presented as the mean $\pm \mathrm{SD} .{ }^{\mathrm{a}} \mathrm{P}<0.05$ vs. model; ${ }^{\mathrm{b}} \mathrm{P}<0.05$ vs. SR-BI. CLM, cationic liposomal microbubbles; SR-BI, scavenger receptor B type I; US, ultrasound; TC, total cholesterol; TG, triglyceride; LDL, low density lipoprotein; HDL, high density lipoprotein.

analysis from the rat carotid arteries that were imaged by US. Western blot analysis was performed as described previously (Fig. 1) (17).

In the remaining rats (10/group), serum TC, TG, LDL and HDL levels were measured 2 weeks post-transfection (Fig. 1).

Statistical analysis. All data are presented as the mean \pm SD. Paired t-tests were used to compare differences in the biochemical variables between the control and model groups at baseline. Multiple comparisons among the groups were performed with one-way ANOVA analysis. $\mathrm{P}<0.05$ was considered to indicate a statistically significant difference.

\section{Results}

Particle size and zeta potential. The lyophilized cake of CLMs retained a free powdery appearance (Fig. 2). Rehydrated formulations were clear dispersions with no visible particles. The particle size was $1.17 \pm 0.06 \mu \mathrm{m}$ and the PDI was $0.364 \pm 0.015$. Fig. 3 reveals a representative image of CLMs captured by optical microscopy. The $\mathrm{pH}$ was 7.34 and the Zeta potential value was $8.10 \pm 8.08 \mathrm{mV}$ (Fig. 4).

US contrast imaging of carotid arteries. CLMs were found to markedly enhance carotid artery imaging in all the rats. The echo intensities of normal carotid arteries increased rapidly, peaking $3 \mathrm{sec}$ after injection of the CLMs. In addition, enhanced normal carotid artery echo intensity was maintained for a longer duration of time (>3 min; Fig. 5).

In vivo gene delivery. As demonstrated in Fig. 6, a marked increase in SR-BI protein was observed 2 days after transfection in the carotid arteries in the SR-BI + US/CLM group, whereas no significant increase in SR-BI protein was detected in the carotid arteries from the SR-BI group $(\mathrm{P}>0.05$, compared with the model group).

Biochemical assay. No significant difference in the serum lipid levels between the control and model groups was found at baseline. Following 8 weeks of a high cholesterol diet, serum TC, TG, LDL and HDL levels were 2.91 $\pm 0.05,1.94 \pm 0.10$, $0.72 \pm 0.09$ and $0.48 \pm 0.02 \mathrm{mmol} / 1$, respectively (Table I). After 2 weeks of SR-BI gene transfection (combined CLMs and US), the plasma lipid levels of the hypolipidemic rats were significantly reduced compared with the untreated rats. However, no significant difference in LDL levels was identified between the model and SR-BI + US/CLM groups. In addition, no significant change in TC levels was observed in the SR-BI group compared with the SR-BI + US/CLM group (Table II).

\section{Discussion}

A number of previous studies have indicated that enhanced transfection with US and microbubble vehicles is caused by the extravascular deposition of DNA, which results from the disruption and dispersion of the microbubble shell (8-12). In the present study, CLMs were developed using the thin-film dispersion method as a chemical carrier for SR-BI DNA delivery. When combined, US and CLMs were found to function synergistically to increase SR-BI DNA transfection. Although a significant improvement in gene expression activity and a longer metabolic half-life were achieved, the long-term preservation of the pcDNA/CLM solution remained a concern.

Due to several physical and chemical factors, freshly prepared formulations demonstrated a loss of transfection activity after 1 week of storage at room temperature. Lyophilization has been employed widely to produce highly 
A
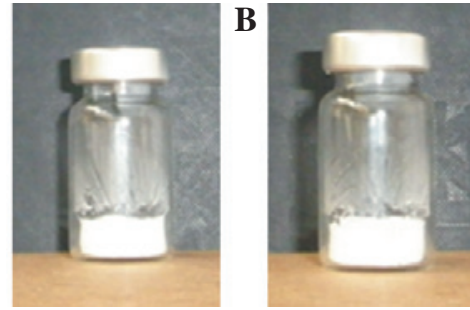

Figure 2. Appearance of the lyophilized cake of the cationic liposomal microbubbles (A) immediately following freeze drying and (B) following 3 months of storage at $25^{\circ} \mathrm{C}$.

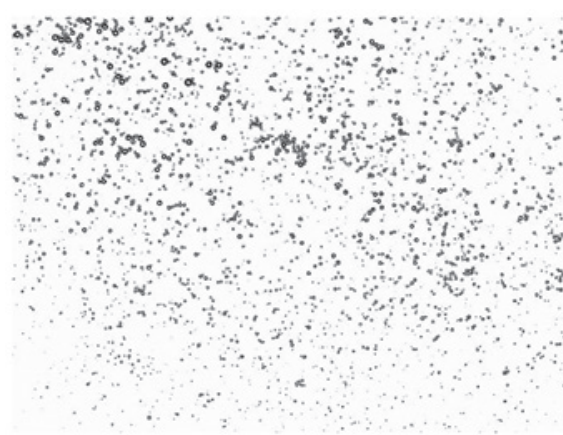

Figure 3. Image of the cationic liposomal microbubbles captured by optica microscopy (magnification, $\mathrm{x} 40$ )

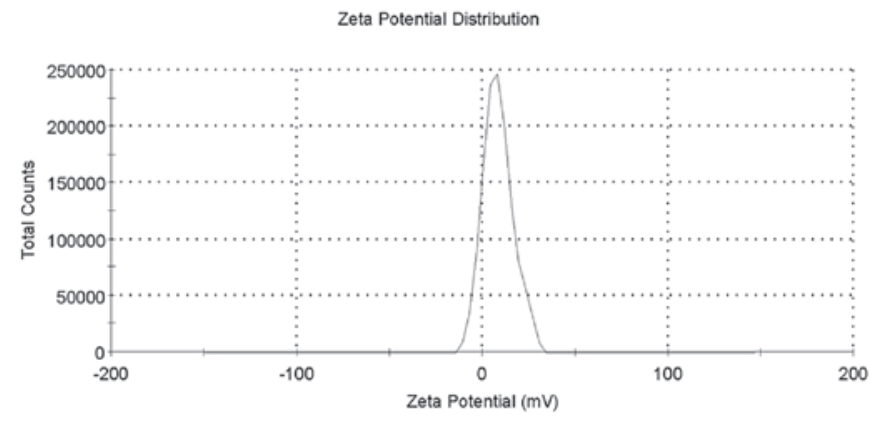

Figure 4. Zeta potential distribution of the cationic liposomal microbubbles.

stable pharmaceutical products $(18,19)$. More recently, lyophilization has also been investigated as a practical technique to produce non-viral vectors with long-term stability (20-22). Lyophilization significantly improved the physical stability of CLMs compared with liquid formulations. In our study, CLMs were developed using the sonication-lyophilization method. The formulations are suitable for long-term preservation at room temperature and microbubbles are able to be used on a large scale.

The consumption of a high-cholesterol diet contributes to cardiovascular disease, which is caused by atherosclerosis, hyperlipidemia and abnormal lipid metabolism. Vitamin D has been widely used to establish animal models of atherosclerosis or calcific vasculopathy. There is a marked correlation between vitamin $\mathrm{D}$ and atherosclerotic calcification or aortic medial calcification, which are likely to involve multiple mechanisms within the bone-vascular-renal endocrine axis (23). In animal models, vitamin D treatment is markedly associated with increased arterial calcification $(24,25)$. Based

A

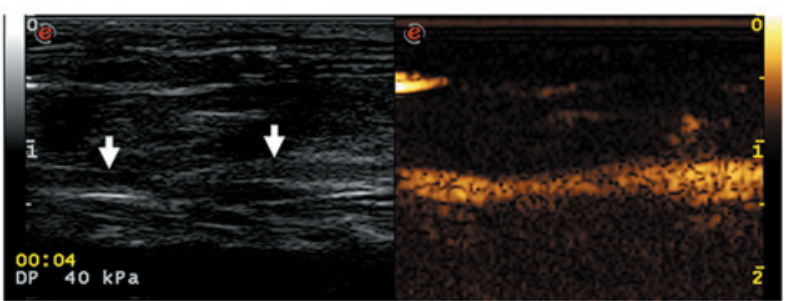

B

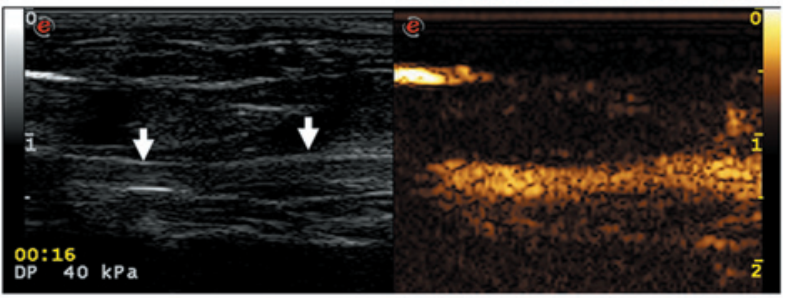

C

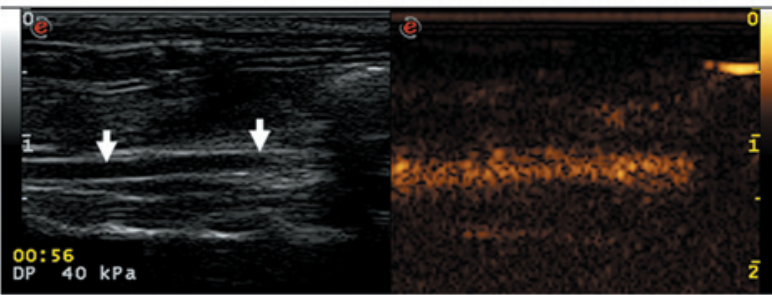

D

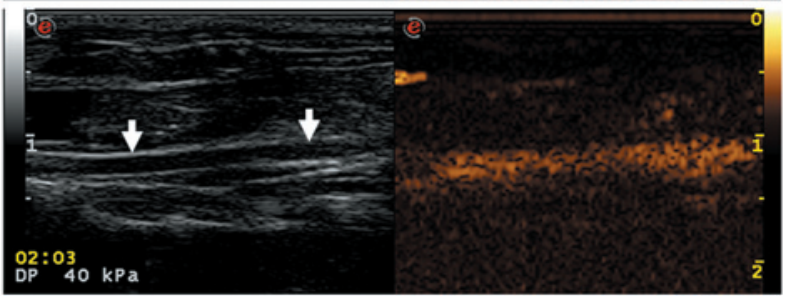

Figure 5. Contrast-enhanced imaging of the carotid artery following intravenous injection of CLMs (arrow indicates the carotid artery). Images of the carotid artery (A) 4, (B) 16, (C) 56 and (D) $123 \mathrm{sec}$ after injection of CLMs. CLMs, cationic liposomal microbubbles.

\section{Scavenger Receptor SR-BI}

GAPDH

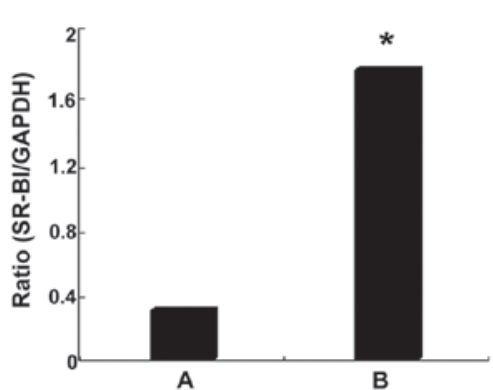

Figure 6. Expression of SR-BI in each group, as determined by western blot analysis. A, SR-BI; and B, SR-BI + US/CLM. GAPDH served as the reference. Bar graphs demonstrate the ratio of SR-BI to GAPDH and data are presented as the mean $\pm \mathrm{SD}(\mathrm{n}=10)$. $\mathrm{P}<0.05$ vs. the SR-BI group. SR-BI, scavenger receptor B type I; US, ultrasound; CLMs, cationic liposomal microbubbles.

on these studies, a rat model of hyperlipidemia was established by administering rats with vitamin D3 and a high-cholesterol diet. However, blood lipid analysis results in the model group were inconsistent with a previous study (26). Differences in the 
components of the high-fat diet and the use of intraperitoneal injection to administer vitamin D3 in the present study may account for this discrepancy.

The marked correlation between HDL levels and the incidence of cardiovascular disease is well established (27). SR-BI was the first HDL receptor to be identified (28), mediating the high affinity binding of HDL by facilitating the bidirectional flux of cholesterol across the plasma membrane. SR-BI has been reported to be significantly expressed in cells involved in reverse cholesterol transport, including atherosclerotic plaque macrophages and hepatocytes (29-31). To examine the feasibility of gene therapy, serum TC, TG, LDL and HDL levels were analyzed in rats 2 weeks post-transfection with SR-BI. Treatment with SR-BI DNA by combining CLMs and US was identified to significantly decrease plasma lipid levels; however, no significant difference in the LDL levels between the model and SR-BI + US/CLM groups was observed (Table II). The lack of significance between LDL levels in the two groups is most likely due to the small sample size. In addition, the main limitation of the present study was that histological identification was not used. Further studies are required to overcome these limitations.

Results of the current study highlight a novel therapeutic strategy which utilizes SR-BI DNA delivered by combining CLMs and US and indicate that this strategy may protect against hypercholesterolemia.

\section{Acknowledgements}

The present study was supported grants from the National Natural Science Foundation of China (no. 30970794) and the Natural Science Foundation of Shanghai Science and Technology Commission (no. 09ZR1424300).

\section{References}

1. Suanarunsawat T, Devakul Na Ayutthaya W, Songsak T, Thirawarapan S and Poungshompoo S: Antioxidant activity and lipid-lowering effect of essential oils extracted from Ocimum sanctum L. leaves in rats fed with a high cholesterol diet. J Clin Biochem Nutr 46: 52-59, 2010.

2. Parthasarathy S, Barnett J and Fong LG: High-density lipoprotein inhibits the oxidative modification of low-density lipoprotein. Biochim Biophys Acta 1044: 275-283, 1990.

3. Fielding CJ and Fielding PE: Molecular physiology of reverse cholesterol transport. J Lipid Res 36: 211-228, 1995.

4. Oram JF and Yokoyama S: Apolipoprotein-mediated removal of cellular cholesterol and phospholipids. J Lipid Res 37: 2473-2491, 1996.

5. Han J, Parsons M, Zhou X, Nicholson AC, Gotto AM Jr and Hajjar DP: Functional interplay between the macrophage scavenger receptor class B type I and pitavastatin (NK-104). Circulation 110: 3472-3479, 2004.

6. Hakkarainen T, Hemminki A, Curiel DT and Wahlfors J: A conditionally replicative adenovirus that codes for a TK-GFP fusion protein (Ad5Delta24TK-GFP) for evaluation of the potency of oncolytic virotherapy combined with molecular chemotherapy. Int J Mol Med 18: 751-759, 2006.

7. Iwamoto HS, Trapnell BC, McConnell CJ, Daugherty C and Whitsett JA: Pulmonary inflammation associated with repeated, prenatal exposure to an E1, E3-deleted adenoviral vector in sheep. Gene Ther 6: 98-106, 1999.

8. Taniyama Y, Tachibana K, Hiraoka K, et al: Development of safe and efficient novel nonviral gene transfer using ultrasound: enhancement of transfection efficiency of naked plasmid DNA in skeletal muscle. Gene Ther 9: 372-380, 2002.
9. Taniyama Y, Tachibana K, Hiraoka K, et al: Local delivery of plasmid DNA into rat carotid artery using ultrasound. Circulation 105: 1233-1239, 2002.

10. Christiansen JP, French BA, Klibanov AL, Kaul S and Lindner JR: Targeted tissue transfection with ultrasound destruction of plasmid-bearing cationic microbubbles. Ultrasound Med Biol 29: 1759-1767, 2003.

11. Huang SL: Liposomes in ultrasonic drug and gene delivery. Adv Drug Deliv Rev 60: 1167-1176, 2008.

12. Suzuki R, Takizawa T, Negishi Y, et al: Gene delivery by combination of novel liposomal bubbles with perfluoropropane and ultrasound. J Control Release 117: 130-136, 2007.

13. Lindner JR: Microbubbles in medical imaging: current applications and future directions. Nat Rev Drug Discov 3: 527-532, 2004.

14. Li T, Tachibana K, Kuroki M and Kuroki M: Gene transfer with echo-enhanced contrast agents: comparison between Albunex, Optison and Levovist in mice - initial results. Radiology 229: 423-428, 2003

15. Ilies MA, Seitz WA and Balaban AT: Cationic lipids in gene delivery: principles, vector design and therapeutical applications. Curr Pharm Des 8: 2441-2473, 2002.

16. Hirko A, Tang F and Hughes JA: Cationic lipid vectors for plasmid DNA delivery. Curr Med Chem 10: 1185-1193, 2003.

17. Ritsch A, Tancevski I, Schgoer W, et al: Molecular characterization of rabbit scavenger receptor class B types I and II: portal to central vein gradient of expression in the liver. J Lipid Res 45: 214-222, 2004

18. Wang W: Lyophilization and development of solid protein pharmaceuticals. Int J Pharm 203: 1-60, 2000.

19. Franks F: Freeze-drying of bioproducts: putting principles into practice. Eur J Pharm Biopharm 45: 221-229, 1998.

20. Molina MD, Armstrong TK, Zhang Y, Patel MM, Lentz YK and Anchordoquy TJ: The stability of lyophilized lipid/DNA complexes during prolonged storage. J Pharm Sci 93: 2259-2273, 2004.

21. del Pozo-Rodríguez A, Solinís MA, Gascón AR and Pedraz JL: Short- and long-term stability study of lyophilized solid lipid nanoparticles for gene therapy. Eur J Pharm Biopharm 71: 181-189, 2009.

22. Kasper JC, Schaffert D, Ogris M, Wagner E and Friess W: Development of a lyophilized plasmid/LPEI polyplex formulation with long-term stability - A step closer from promising technology to application. J Control Release 151: 246-255, 2011.

23. Hsu JJ, Tintut Y and Demer LL: Vitamin D and osteogenic differentiation in the artery wall. Clin J Am Soc Nephrol 3: 1542-1547, 2008

24. Hung CR, Chen WH and Wang PS: Protective effect of lysozyme chloride on gastric oxidative stress and hemorrhagic ulcers in severe atherosclerotic rats. Med Sci Monit 13: BR271-BR279, 2007.

25. Wu Y, Li J, Wang J, Si Q, Zhang J, Jiang Y and Chu L: Anti-atherogenic effects of centipede acidic protein in rats fed an atherogenic diet. J Ethnopharmacol 122: 509-516, 2009.

26. Wan CW, Wong CN, Pin WK, et al: Chlorogenic acid exhibits cholesterol lowering and fatty liver attenuating properties by up-regulating the gene expression of PPAR- $\alpha$ in hypercholesterolemic rats induced with a high-cholesterol diet. Phytother Res 2012: Jun 6, 2012 (Epub ahead of print).

27. Hong SC, Zhao SP and Wu ZH: Effect of probucol on HDL metabolism and class B type I scavenger receptor (SR-BI) expression in the liver of hypercholesterolemic rabbits. Int J Cardiol 115: 29-35, 2007.

28. Acton S, Rigotti A, Landschulz KT, Xu S, Hobbs HH and Krieger M: Identification of scavenger receptor SR-BI as a high density lipoprotein receptor. Science 271: 518-520, 1996.

29. Hirano K, Yamashita S, Nakagawa Y, et al: Expression of human scavenger receptor class B type I in cultured human monocyte-derived macrophages and atherosclerotic lesions. Circ Res 85: 108-116, 1999.

30. Trigatti BL, Krieger M and Rigotti A: Influence of the HDL receptor SR-BI on lipoprotein metabolism and atherosclerosis. Arterioscler Thromb Vasc Biol 23: 1732-1738, 2003.

31. Chinetti G, Gbaguidi FG, Griglio S, et al: CLA-1/SR-BI is expressed in atherosclerotic lesion macrophages and regulated by activators of peroxisome proliferator-activated receptors. Circulation 101: 2411-2417, 2000. 